\title{
CANCER GENOMICS
}

\section{A nucleosome footprint reveals the source of cfDNA}

A group from the University of Washington have used cell-free DNA (cfDNA) from human plasma to map genome-wide in vivo nucleosome occupancy, enabling them in some cases to determine the cell type of origin of circulating cfDNA from cancer patients. cfDNA-based screening is increasingly used in oncology and prenatal medicine, but its applications are limited by a reliance on sequence-level differences to distinguish between contributing cell types.

Previous observations of cfDNA fragment size distributions had noted peaks corresponding to DNA associated with nucleosomes ( $147 \mathrm{bp})$. This finding suggests that DNA is protected from nuclease digestion through its association with a nucleosome core particle. The investigators therefore hypothesized that the fragmentation patterns of an individual's cfDNA might contain evidence of the epigenetic profile of the cells of origin. Such a footprint could potentially be used to determine the contributing cell types in the absence of genotypic differences.
To investigate their hypothesis, the group deep-sequenced cfDNA from the plasma of healthy individuals. Based on the expectation that fragment endpoints should cluster next to nucleosome boundaries and should be depleted at sites of nucleosome occupancy, they then generated genome-wide maps of in vivo nucleosome protection.

The inferred nucleosome spacing correlated with the cellular expression of genes, suggesting that nucleosome occupancy could be used to determine the tissue of origin of cfDNA. Analysis based on this correlation, and using expression data sets from 76 human cell lines and primary tissues, identified lymphoid, myeloid and bone marrow tissue as the primary sources of cfDNA, consistent with haematopoietic cell apoptosis as the main source of cfDNA in healthy individuals.

To explore whether the footprint of non-haematopoietic cells could be detected in the cfDNA of non-healthy individuals, the group sequenced cfDNA from the plasma of five individuals with stage IV cancer. In contrast to the analysis of cfDNA from healthy individuals, many of the cell lines detected as main contributors to cfDNA from cancer patients represented non-haematopoietic lineages,

z and in some cases these matched the cancer type of the individual. The authors suggest that the lack of alignment of some cancers with a specific cell line might be explained by a lack of representation in the 76 expression data sets used.

"The study has implications both for our basic understanding of the origins of cell-free DNA and for ways we might use cell-free DNA in the clinic to detect an increasing number of physiological conditions and diseases," explains Matthew Snyder, co-first author on the paper. "Our method does not rely on sequence-level differences, opening up a new approach for non-invasive screening," comments co-first author Martin Kircher. "We hope to use these findings to inform the development of next-generation diagnostics aimed not only at cancer and pregnancy but for a wider range of conditions," Kircher concludes.

Denise Waldron

ORIGINAL ARTICLE Snyder, M. W. et al. Cell-free DNA comprises an in vivo nucleosome footprint that informs its tissues-of-origin. Cell 164, 57-68 (2016) 